\title{
Embedded Real-Time ECG Delineation Methods: a Comparative Evaluation
}

\author{
Rubén Braojos \\ Giovanni Ansaloni \\ David Atienza \\ Embedded Systems Laboratory \\ École Polytechnique Fédérale de Lausanne, Switzerland \\ Email: $\{$ ruben.braojoslopez $\},\{$ giovanni.ansaloni\}, \\ \{david.atienza\}@epfl.ch
}

\author{
Francisco J. Rincón \\ Departamento de Arquitectura de Computadores y Automtica \\ Universidad Complutense de Madrid, Spain \\ Email: francisco.rincon@fdi.ucm.es
}

\begin{abstract}
Wireless sensor nodes (WSNs) have recently evolved to include a fair amount of computational power, so that advanced signal processing algorithms can now be embedded even in these extremely low-power platforms. An increasingly successful field of application of WSNs is tele-healthcare, which enables continuous monitoring of subjects, even outside a medical environment. In particular, the design of solutions for automated and remote electrocardiogram (ECG) analysis has attracted considerable research interest in recent years, and different algorithms for delineation of normal and pathological heart rhythms have been proposed. In this paper, some of the most promising techniques for filtering and delineation of ECG signals are explored and comparatively evaluated, describing their implementation on the state-of-the-art IcyHeart WSN. The goal of this paper is to explore the trade-offs implied in the different settings and the impact of design choices for implementing "smart" WSNs dedicated to monitoring ECG bio-signals.
\end{abstract}

Index Terms-Embedded Systems, Bio-medical Signal Processing, Wireless Sensor Networks, ECG Delineation, ECG Filtering.

\section{INTRODUCTION}

The aging of world population, coupled with others factors such as the predominance of unhealthy lifestyles, is enlarging the impact of cardiovascular disorders, which now represent the most common global cause of death [1]. Supervising patients affected by cardiopathy is putting an increasing pressure over health care systems.

Electrocardiograms (ECGs), which measure the electrical activity of the heart, are the primary instruments for monitoring the heart activity and for early detection of heart pathologies. A breakthrough in the practice of ECGs recording and analysis has been possible thanks to Wireless ECG Sensor Nodes (WESNs) [2][3]: miniaturized, wearable ECG devices that are able to wirelessly transmit relevant data, allowing the continuous and autonomous monitoring of subjects.

The continuous progress in semiconductor technology has enabled the emergence of "smart" WESNs that, in addition to acquisition and transmission, perform an automated diagnosis by interpreting ECG data [4][5]. Smart WESNs applications

The research illustrated in this paper has been supported by the IcyHeart European project (FP7, capacities. Proj. num. 286130.) usually implement algorithms to filter acquired signals, delineate individual heart beats to retrieve their characteristics and classify them to detect pathologies. The first two steps (filtering and delineation), focus of this paper, have the most challenging real-time constraints, because they deal with the manipulation and analysis of digital signals [6][7][8], as opposed to parameters of whole heart beats.

While proposals are described in the literature to implement embedded delineation and filtering [9][10], a comparative evaluation of different methods is still lacking, and this paper aims at filling this gap. In this work we specifically target WESN solutions, only considering methods that can be implemented on these resource-constrained platforms, which usually only support integer arithmetic and present a small memory size.

While delineation could be conceptually performed on raw signals, we do not consider this option in this work, as realworld acquisitions are always corrupted by both low-frequency noise sources (caused by subjects' respiration and perspiration) and high-frequency ones (due to muscular contractions or misplacements of electrodes), mandating a filtering phase [6].

Filtering is here intended in broad terms, comprising all operations that can increase the signal quality of an acquired ECG. In this context, an important design choice whose implication is here investigated is between adapting a highfrequency sampling of a single signal or fusing data from multiple sensors (leads) sampled at a lower frequency. Moreover, some of the proposed data fusion algorithms in the literature,

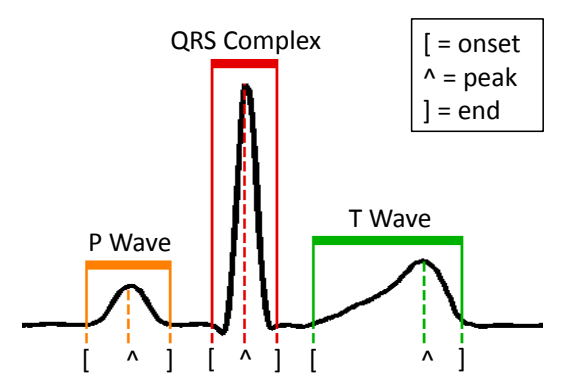

Fig. 1. Delineated normal sinus beat. 


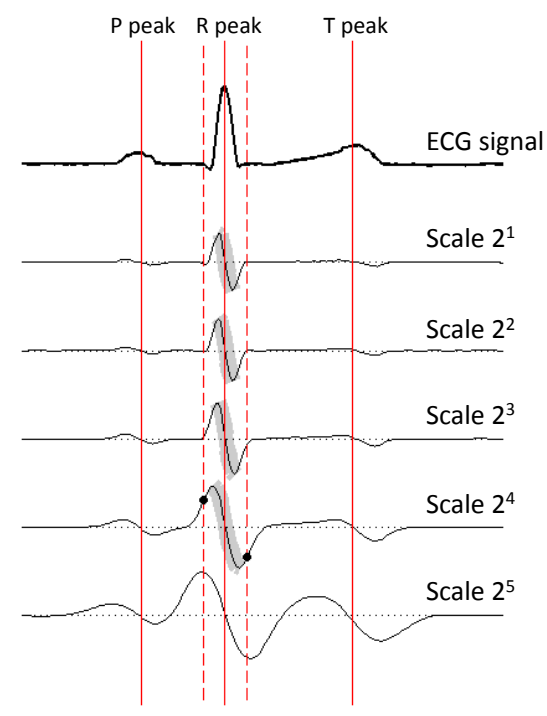

Fig. 2. DWT decomposition of an ECG heart beat. Maximum-minimun intervals examined to find the $\mathrm{R}$ peak are highlighted in gray.

like the iterative WT method presented in [11] or the derivative based described in [12], are too computationally demanding to be executed on a WESN platform. The simpler solution employed in this study [13], is then to consider the RMS (Root Mean Square) combination among synchronous samples from different leads.

In addition, low-frequency noise components cannot be mitigated by high-frequency sampling or data fusion. To filter them, specific algorithms to eliminate baseline wandering have to be applied; in the paper, we explore the effectiveness of two solutions, based on cubic spline interpolation [14] and morphological operators [8].

Filtered signals are then analyzed by a delineation pass to find the fiducial points of each heart beat, corresponding to the onset, peak and end of its characteristic waves: P, QRS complex and T (Figure 1); abnormalities in the length of ECG waves are important markers of different heart conditions.

Many approaches have been proposed to automate ECG delineation. Some of them (such as methods based on lowpass differentiation [6], neural netwoks [15] or hidden Markov models [16]) present a high computational complexity, so that they cannot be adopted in WESNs. Thus, the possible options considered in this study are based on Digital Wavelet Transforms (DWTs) [17] [18] and Multiscale Morphological Derivatives (MMDs) [7] since, while also conceived as off-line algorithms, these approaches can be optimized for real-time embedded delineation [9] [10].

Finally, the information about the delineated points can either be processed on the node or sent trough a wireless link to a remote station, with or without the support of the acquired signal. As it has been already shown in recent works, it is worth mentioning that by transmitting only information on the heart beat waveforms, energy efficiency can be considerably increased by minimizing communication on the power-hungry wireless link [10].

Overall, in this paper we explore the design space offered by the different possible embedded filtering and delineation choices when processing in real-time a variety of normal and pathological ECG recordings, retrieved from the Common Standards for Electrocardiography (CSE) database [19]. Performance is assessed along three dimensions: delineation accuracy, robustness against noise and computational requirements of the implementations.

We have selected as representative target WESN platform for our exploration the IcyHeart System-on-Chip [20], which embeds a 32-bit low-power processor (running at $6 \mathrm{MHz}$ and having $96 \mathrm{~KB}$ of memory), a multi-channel analog-to-digital converter and a wireless transmission module. IcyHeart is the first SoC presenting these three components integrated on the same silicon die.

The manuscript proceeds as follows: in Section II and III, the considered delineation and filtering methods, respectively, are described. In Section IV experimental results assessing their performance are presented. Our concluding remarks are summarized in Section V.

\section{EMBEDDED DELINEATION METHODS}

\section{A. Wavelet-based delineation}

DWT delineation considers a decomposition of acquired signals in five dyadic scales, which can be efficiently computed using a filter bank composed by low- and high- pass FIR filters. Scales represent derivatives of smoothed versions of the input ECG signals, as exemplified in Figure 2. To ensure timeinvariance among different scales, the filter impulse response is interpolated using the algorithme á trous method, illustrated in [21].

Because the different characteristic waves of beats present distinct frequency contents, their fiducial points are retrieved at different scales, the QRS complex being reflected in scales $2^{1}$ to $2^{4}$, while $\mathrm{P}$ and $\mathrm{T}$ waves presents their major components in scales $2^{4}$ and $2^{5}$.

As scales are computed, the DWT delineator searches for maximum-moduli points at the different scales, reflecting points of maximum slope in the acquired signals. The $\mathrm{R}$ peak is identified as the zero-crossing point at scale $2^{1}$ in-between tuples of maximum moduli with different signs across scales from $2^{1}$ to $2^{4}$. Dynamic thresholding is performed to reject maximum moduli with small absolute values.

QRS onset is identified at scale $2^{4}$ by a back-search for the point where its absolute value becomes smaller than one-fourth of the peak associated with the wave. Similarly, the QRS end is retrieved by a forward search for the point where scale $2^{4}$ becomes smaller than three-fourths of its maximum absolute value.

Focusing on search windows before and after the QRS complex, $\mathrm{P}$ and $\mathrm{T}$ peaks are identified as the zero-crossing points at scale $2^{3}$ between two maximum moduli either at scale $2^{4}$ or, if not such tuple is found, at scale $2^{5}$. Even for $\mathrm{P}$ and $\mathrm{T}$ waves, dynamic thresholding is employed to filter 


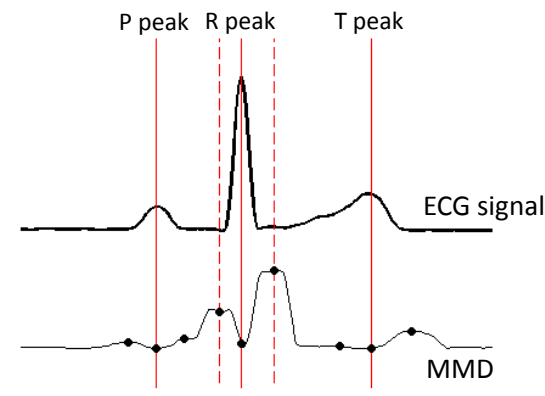

Fig. 3. Acquired signal (top) and its MMD-transformed version (bottom).

maximum moduli. Calculation of the onset and end of $\mathrm{P}$ and $\mathrm{T}$ waves is then similar to the $\mathrm{QRS}$ case.

\section{B. MMD-based delineation}

The morphological derivative $M_{f}^{d}$ of a discrete signal $f$ : $D \subset \mathbb{R} \rightarrow \mathbb{R}$ at scale $s$ is defined as:

$$
M_{f}^{d}(x)=\frac{\left(f \oplus g_{s}\right)(x)+\left(f \ominus g_{s}\right)(x)-2 f(x)}{s}
$$

where $g_{s}: G_{s} \subset \mathbb{R} \rightarrow \mathbb{R}$ is a structuring element of length $s$ and the $\oplus$ (dilation) and $\ominus$ (erosion) operations are defined as:

$$
\begin{aligned}
& \text { Dilation: }\left(f \oplus g_{s}\right)(x)=\max _{t \in\left(G_{s} \cap D_{x}\right)}\left\{f(x-t)+g_{s}(t)\right\} \\
& \text { Erosion : }\left(f \ominus g_{s}\right)(x)=\min _{t \in\left(G_{s} \cap D_{x}\right)}\left\{f(x+t)-g_{s}(t)\right\}
\end{aligned}
$$

Where $D_{x}$ is $D$ translated by $x$.

If a flat structuring element is chosen, computing the morphological derivative of $f$ can be performed by sliding a window of size $s$ over the signal, and calculating the maximum and minimum of the signal as well as its value in the central point of the window:

$$
M_{f}^{d}(x)=\frac{\max \{f(t)\}_{t \in I}+\min \{f(t)\}_{t \in I}-2 f(x)}{s}
$$

where $I=[x-s, x+s]$.

As Figure 3 shows, the morphological transformation translates peaks on the input signal in pits on the transformed one, while peaks or sudden change in slope of the transformed signal highlights onsets or ends of waves in the input signal.

A search on the MMD transform for a negative value exceeding a dynamically-adjusted threshold retrieves the peak of the $\mathrm{R}$ wave; peaks (or sudden change in slope) around it retrieve the onset and end of the QRS complex. Before and after the found QRS complex, tuples of zero-crossing points mark the presence of the $\mathrm{P}$ and $\mathrm{T}$ waves respectively. $\mathrm{P}$ and $\mathrm{T}$ peaks are identified by the minimum value in-between the crossing points, while their onset and end by the maximum values before and after the crossing points.

\section{MULTI-LEAD COMBINATION}

WESN devices usually acquire multiple signals concurrently, giving the opportunity to increase the delineation performance by fusing acquired data sampled in different leads. In this paper, we adopted RMS (Root Mean Square) combination of synchronous samples of different channels:

$$
x(t)_{R M S}=\sqrt{\frac{1}{N} \sum_{i=1}^{N}\left(x_{i}(t)^{2}\right)}
$$

The example in Figure 4 showcases its benefits: as this figure shows, lead I presents a small $\mathrm{P}$ wave, while lead III is noisy and has a low $\mathrm{T}$ wave. However, their combination has a higher quality compared to each lead in isolation.

To properly RMS-combine signals, they must be firstly centered on the iso-electric line, eliminating low-frequency baseline wandering. The two investigated approaches to implement this step, based on cubic spline interpolation and morphological operators are described hereafter.

\section{A. Spline filtering $(S F)$}

This method assumes that several time intervals of an ECG are silent, i.e. they are devoid of any heart activity [22]. One such segment is the interval between the $\mathrm{P}$ and the $\mathrm{Q}$ waves. Stemming from this observation, it is then possible, by placing "knots" on the PQ segment and fitting a cubic polynomial in successive triplets of knots, to estimate the baseline. For each interval between beats $[i, i+1]$, the knots identified for the beats $i, i+1$ and $i+2$ are considered to derive the estimated baseline.

Determining the knots position requires an estimation of the QRS complex onset, which in our implementation is realized employing a simplified DWT delineator on each lead.

\section{B. Morphological filtering $(M F)$}

The erosion and dilation operators introduced in Section II-B are here used in a different scenario, to perform filtering operations. Hence, they are combined to generate the opening and closing functions. Opening (o) of a function $f$ using a structuring element $g_{s}$ is defined as:

$f \circ g_{s}=\left(f \ominus g_{s}\right) \oplus g_{s}$

If $g_{s}$ is flat, this operations removes from $f$ peaks of length smaller than $s$. Dually, closing $(\bullet)$, defined as

$f \bullet g_{s}=\left(f \oplus g_{s}\right) \ominus g_{s}$

removes pits of length smaller than $s$. By employing structuring elements of length greater than the longest ECG
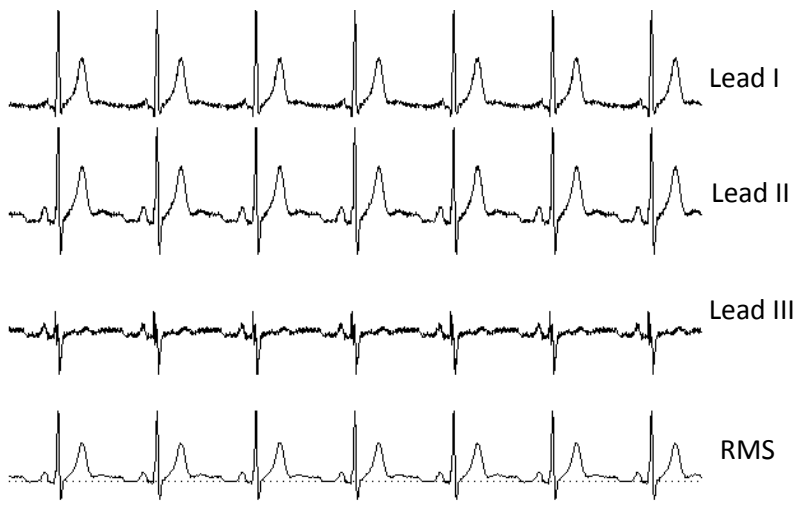

Fig. 4. Three-leads acquisition and corresponding RMS-combined signal. 


\begin{tabular}{r|cc|cc|cc|cc} 
& \multicolumn{2}{|c|}{ MF+MMD } & \multicolumn{2}{c|}{ MF+DWT } & \multicolumn{2}{c|}{ SF+MMD } & \multicolumn{2}{c}{ SF+DWT } \\
\hline & Sen & $P+$ & Sen & $P+$ & Sen & $P+$ & Sen & $P+$ \\
\hline P onset & 0.71 & 0.78 & 0.78 & 0.86 & 0.57 & 0.68 & 0.72 & 0.84 \\
P end & 0.68 & 0.71 & 0.79 & 0.82 & 0.64 & 0.73 & 0.72 & 0.81 \\
QRS onset & 0.94 & 0.95 & 0.98 & 0.98 & 0.86 & 0.96 & 0.97 & 0.98 \\
QRS end & 0.94 & 0.94 & 0.97 & 0.97 & 0.88 & 0.86 & 0.96 & 0.97 \\
T end & 0.71 & 0.75 & 0.78 & 0.86 & 0.37 & 0.46 & 0.82 & 0.89
\end{tabular}

TABLE I

SENSITIVITY AND POSITIVE PREDICTIVITY OF DELINEATED FIDUCIAL POINTS (SINGLE-LEAD, 250 SAMPLES/SEC).

wave (typically, the $\mathrm{T}$ wave), it is then possible to derive the appropriate baseline, that can then be subtracted from the acquired signal.

It is worth mentioning that morphological methods can be used also to filter high-frequency muscular noise. In this case, structuring elements shorter than the shortest ECG wave are instead employed to perform the opening and closing operations.

\section{EXPERIMENTAL EVALUATION}

We evaluated four different combinations of filtering and delineation methods performing automated retrieval of the heart beats fiducial points: highly optimized implementations of the DWT- and MMD- based delineation algorithms were coupled with either morphological or cubic spline baseline wandering elimination.

To comprehensively evaluate their performance, five rounds of experiments were conducted. Firstly, we measured their accuracy on single lead recordings sampled at 250 samples/sec (Section IV-A). We then explored the impact of fusing recordings of two or three leads (Section IV-B) and of increasing the sampling frequency to $500 \mathrm{samples} / \mathrm{sec}$ (Section IV-C).

We also evaluated the algorithms robustness, by artificially adding different levels of noise to the recordings; related results are discussed in Section IV-D. Finally, resource usage (computing effort and memory footprint) is an important factor when considering applications for WESNs. Thus, we present comparative data in this regard in Section IV-E.

In our experiments we have retrieved the evaluated ECG recordings from the DS1 dataset of the Common Standards for Electrocardiography (CSE) [19] ECG database. This database contains challenging samples both of healthy patients and of subjects presenting a wide range of pathologies (e.g.: bundle blocks, myocardial infarctions, atrial flutter...).

\section{A. Delineation accuracy}

To quantitatively compare the accuracy of the different solutions, we associated the manually annotated fiducial points provided by the database distribution (comprising the onset and end of the $\mathrm{P}$ wave, the onset and end of the QRS complex and the end of the $T$ wave) to the ones that are automatically retrieved using different filtering/delineation applications.

Table I shows the sensitivity $(S e)$ and positive predictivity $(P+)$, measured when applying each method. $S e$ and $P+$ are defined as

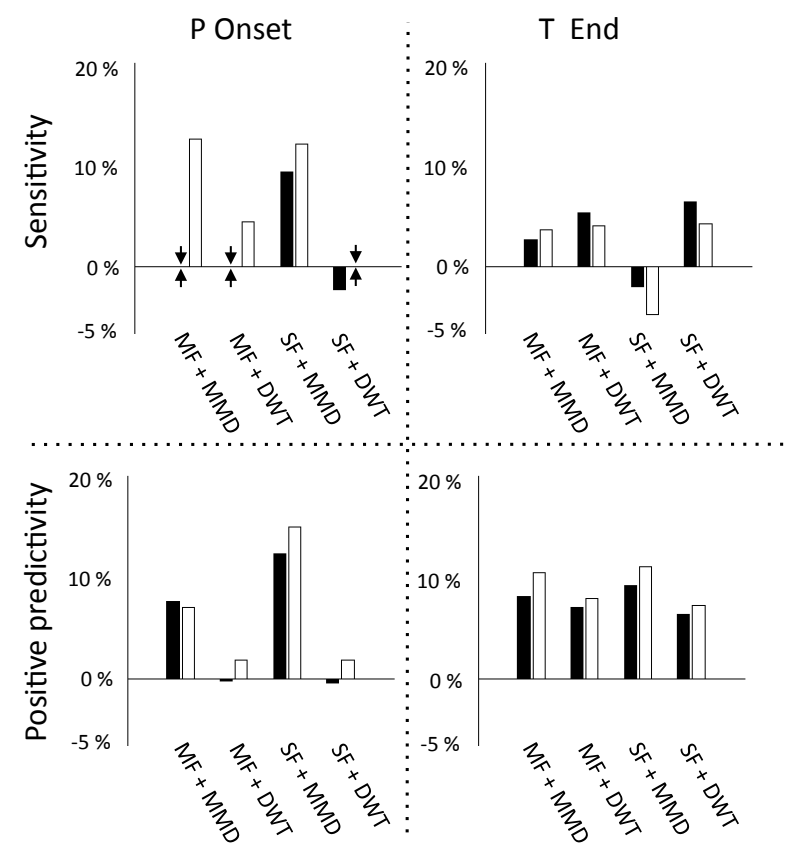

Fig. 5. Relative improvement in sensitivity and positive predictivity of twoand three- leads delineation with respect to single-lead delineation. Black $=2$ leads, White $=3$ leads.

$$
S e=\frac{T P}{T P+F N}, \quad P+=\frac{T P}{T P+F P}
$$

An automatically delineated point represents a True Positive $(T P)$ if a corresponding manual annotation is present in a time interval no bigger than $80 \mathrm{~ms}$; if such annotation does not exist, it is instead considered a False Positive $(F P)$. If a manual annotation has no corresponding automatic one in a $80 \mathrm{~ms}$ neighborhood, it is counted as a False Negative $(F N)^{1}$.

A first observation from Table I is that some fiducial points are more challenging than others; in particular, the QRS complex is easier to delineate thanks to the sharpness and the magnitude of its characteristic shape. All methods exhibit therefore good predictivity and sensitivity when identifying its onset and end.

On the other hand, $\mathrm{P}$ waves are smaller, and their fiducial points can be easily masked by background noise. The end of the $\mathrm{T}$ wave is also hard to detect, being usually smooth and not always precisely defined. In these cases, results among different methods are very variable with the combination of spline filtering and MMD transform performing far worse than the other three combinations.

\section{B. Using single-vs. multi- lead delineation}

Figure 5 reports the relative change in sensitivity and predictivity with respect to single-lead when either two or three leads are RMS-combined. For conciseness, only data referring

\footnotetext{
${ }^{1}$ Sensitivity indicates the ratio of manually annotated points that have a corresponding automatic annotation. Positive predictivity instead reports the proportion of automatically reported fiducial points that have a correspondent manual annotation.
} 
to $\mathrm{P}$ onset and $\mathrm{T}$ end is reported, being the most challenging points.

Even if some outliers are present, in most cases fusing data from different leads resulted in an increased delineation quality. While the increase of more than $15 \%$ in positive predictivity achieved for the $\mathrm{P}$ onset point in the case of spline filtering and MMD delineation is somehow biased by its poor single-lead quality, multi-lead delineation proves beneficial even when single-lead performance is already high, as exemplified by the algorithms employing DWT delineation and morphological filtering, where a predictivity of $93 \%$ could be attained for the $\mathrm{T}$ end point using the RMS combination of three leads.

\section{Influence of sampling frequency}

Results reported in the previous sections consider a sampling rate of 250 samples/sec, instead of the original 500 samples/sec provided by CSE recordings. As figures in Table II shows, this downsampling does not degrade the delineation quality, being the vast majority of the frequency components of the heart beat characteristic waves included in the interval ]0, 100] Hz [22].

Table II reports sensitivity and predictivity of implementations tailored to sampling rates of 250 and 500 samples/sec, respectively. Presented data aggregates sensitivity and positive predictivity measurements for the five considered fiducial points and the different leads configurations.

These results show that, surprisingly, an increased sampling frequency does not necessarily improve delineation performance; in fact, results at 500 samples/sec are slightly worse than those where decimation is applied because a slower sampling frequency smoothes signals, thus canceling part of the noise components.

\section{Robustness against noise}

To further investigate the different implementations, their performance degradation has been investigated when random white noise is added to the recordings. Three settings are considered: (1) noisy single-lead delineation, (2) three-leads delineation with noise added to a single lead, and (3) threeleads delineation with noise added to all three leads. The rationale behind these choices is that noise sources like lead misplacements or muscular movements do not affect all leads with the same magnitude, hence real-world multi-lead acquisitions have noise characteristics in-between the two latter settings.

The data presented in Figure 6 refer to the P onset and T end fiducial points, plotting the predictivity-sensitivity product considering a 20 and $10 \mathrm{~dB}$ signal-to-noise ratio (SNR). These results show that the most robust solutions are the ones based on DWT delineations, particularly when coupled with morphological filtering. Cubic spline filtering combined with morphological delineation performed even in this case the worst, being the least effective, most of all for delineating the $\mathrm{T}$ end fiducial point.

\begin{tabular}{c|c|c|c|c} 
& \multicolumn{2}{|c|}{ Se } & \multicolumn{2}{c}{ P+ } \\
\hline Sampling rate & 250 & 500 & 250 & 500 \\
\hline Method & & & & \\
MF+MMD & 0.81 & 0.76 & 0.86 & 0.84 \\
MF+DWT & 0.86 & 0.78 & 0.90 & 0.87 \\
SF+MMD & 0.67 & 0.62 & 0.77 & 0.75 \\
SF+DWT & 0.84 & 0.68 & 0.90 & 0.84
\end{tabular}

TABLE II

SENSITIVITY AND POSITIVE PREDICTIVITY OF DELINEATED FIDUCIAL POINTS (SINGLE-LEAD, 250 AND 500 SAMPLES/SEC). DATA AGGREGATED ON ALL FIDUCIAL POINTS.

Figure 6 also highlights that multi-lead delineations can resist to higher levels of noise with respect to the corresponding single-lead versions. This holds even when a robust implementation is considered and all leads are affected by noise, with an improvement of as much as $10 \%$ in the sensitivitypredictivity product in the case of morphological filtering and DWT delineation at the $10 \mathrm{~dB}$ SNR level. The robustness of multi-lead delineation solutions (with respect to single-lead) is consistent among all methods, strongly indicating that this feature is beneficial for real-world WESN applications.

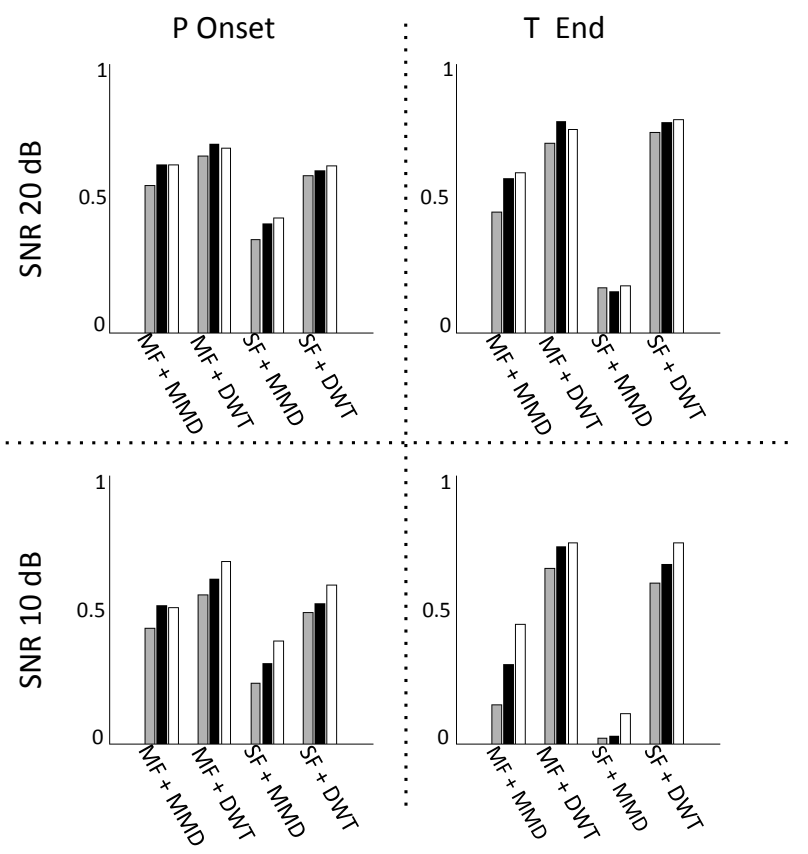

Fig. 6. Predictivity-Sensitivity product for $\mathrm{P}$ onsets and $\mathrm{T}$ ends with different SNR and different number of leads: Grey = Single lead $(1$ noisy), Black = Multi-lead (3 noisy) and White = Multi-lead (1 noisy)

\section{E. Code size and duty cycle evaluation}

To assess the real-time performance of the filtering/delineation algorithms illustrated in Sections II and III, we profiled their execution on the IcyHeart WESNs. Table III reports the resulting memory footprint of the ECG applications and their duty cycle during delineation.

The shown data highlights that data fusion of multiple leads can be performed with a less-than-linear impact on memory size and computation effort, namely 3-leads delineations being 


\begin{tabular}{c|c|c|c|c}
\multicolumn{5}{c}{ Code Size (KB) } \\
\# leads & MF+MMD & MF+DWT & SF+MMD & SF+DWT \\
\hline 1 & 38.29 & 32.42 & 47.51 & 30.40 \\
2 & 42.99 & 37.13 & 58.98 & 40.21 \\
3 & 46.39 & 40.47 & 69.69 & 49.21 \\
\hline \multicolumn{5}{c}{ Duty Cycle(Avg / Max) } \\
\# leads & MF+MMD & MF+DWT & SF+MMD & SF+DWT \\
\hline 1 & $0.30 / 0.47$ & $0.17 / 0.19$ & $0.35 / 0.47$ & $0.24 / 0.31$ \\
2 & $0.43 / 0.53$ & $0.30 / 0.32$ & $0.42 / 0.55$ & $0.32 / 0.39$ \\
3 & $0.58 / 0.67$ & $0.47 / 0.50$ & $0.50 / 0.65$ & $0.38 / 0.48$
\end{tabular}

TABLE III

MEMORY FOOTPRINT (TOP) AND DUTY CYCLE OF FILTERING/DELINEATION APPLICATIONS EXECUTED ON THE ICYHEART PLATFORM (250 SAMPLES/SEC).

on average only $40 \%$ larger and $82 \%$ slower than single-lead ones.

Doubling instead the sampling frequency to 500 samples/sec (which, as stated in Section IV-C has not resulted in higherquality delineations), results in an average increase of $33 \%$ in memory size and $125 \%$ in runtime. The first aspect is reflected by the doubling in size of required buffers, while the second by the linear relation between sampling rate and workload.

Comparing the filtering/delineation application, we clearly conclude that higher workload and memory footprint do not always reflect higher performance. In particular, the combination of morphological filtering and DWT delineation stands out as the least demanding solution from the computational viewpoint, while reaching very good overall results for ECG analysis in real-time.

\section{CONCLUSion}

WESNs embedding advanced real-time signal processing can effectively enable unobtrusive monitoring of patients affected by cardiopathies, improving their quality of life and, at the same time, providing a more effective care.

Real-time ECG delineation, performed directly on "smart" WESNs, is an important stepping stone in this direction. By extracting relevant data (fiducial points of ECG waves) out of acquired cardiac signals, delineation can, on one side, enable further rapid diagnosis of heart conditions, and on the other reduce the amount of data to be wirelessly transmitted, boosting the energy efficiency and ultimately the autonomy of battery-operated WESNs.

Considering the widely different approaches that have been proposed in literature to perform real-time ECG delineation, the choice of a proper implementation is not obvious. In this paper, we have investigated and compared different techniques to filter ECG acquisition and delineate them in real time, while varying design parameters such as the number of acquired leads and the employed sampling frequency. Reported results assess the performance of resulting implementations in terms of accuracy and resistance to noise, as well as code size and computational complexity.

Taking into account the above-mentioned metrics, experimental evidence shows that combining multiple leads at a rather low frequency is a better design choice than sampling a single signal at a higher frequency.
Among the considered combinations of filtering/delineation chains, the RMS combination of three morphologically filtered leads followed by DWT delineation, proved to be the most promising solution for WESN implementations, especially when noise artifacts are considered. The number of combined signals can be reduced when targeting platforms with extremely constrained resources, at the price of a lower accuracy and (especially) resistance to noise.

\section{REFERENCES}

[1] World Health Organization., "Cardiovascular diseases," 2009. [Online]. Available: http://www.who.int/topics/cardiovasculardiseases/

[2] C. Park, P. Chou, Y. Bai, R. Matthews, and A. Hibbs, "An ultra-wearable, wireless, low power ECG monitoring system," BioCAS 2006.

[3] J. Proulx, R. Clifford, S. Sorensen, D.-J. Lee, and J. Archibald, "Development and evaluation of a bluetooth EKG monitoring sensor," CBMS 2006.

[4] A. Burns, B.R. Greene, M.J. McGrath, T.J. O'Shea, B. Kuris, S.M. Ayer, F. Stroiescu and V. Cionca, "SHIMMER A Wireless Sensor Platform for Noninvasive Biomedical Research," IEEE Sensors Journal, 2010.

[5] D. Malan, T. Fulford-Jones, M. Welsh and S. Moulton, "CodeBlue: An Ad Hoc Sensor Network Infrastructure for Emergency Medical Care," International Workshop on Wearable and Implantable Body Sensor Networks, 2004

[6] P. Laguna, R. Jané, and P. Caminal, "Automatic detection of wave boundaries in multilead ECG signals: validation with the CSE database," Computers and Biomedical Research, 1994.

[7] Y. Sun, K. Luk Chan, and S. Muthu Krishnan, "Characteristic wave detection in ECG signal using morphological transform," BMC Cardiovascular Disorders, 2005.

[8] Y. Sun, K. L. Chan, and S. M. Krishnan, "ECG signal conditioning by morphological filtering," Computers in Biology and Medicine, 2002.

[9] F. Rincon, N. Boichat, D. Atienza, and N. Khaled, "Wavelet-based ECG delineation on a wearable embedded sensor platform," BSN 2009.

[10] F. Rincon, J. Recas, N. Khaled, and D. Atienza, "Development and evaluation of multilead wavelet-based ECG delineation algorithms for embedded wireless sensor nodes," IEEE Transactions on Information Technology in Biomedicine, 2011.

[11] R. Almeida, J. Martinez, A. Rocha, and P. Laguna, "Multilead ECG delineation using spatially projected leads from wavelet transform loops," IEEE Transactions on Biomedical Engineering, Aug. 2009.

[12] A. Illanes-Manriquez, "An automatic multi-lead electrocardiogram segmentation algorithm based on abrupt change detection," EMBC 2010.

[13] M. Soria, J. Martinez, and P. Laguna, "A multilead wavelet-based ECG delineator based on the rms signal," CinC 2006.

[14] C. Meyer and H. Keiser, "Electrocardiogram baseline noise estimation and removal using cubic splines and state-space computation techniques," Computers and Biomedical Research, 1977.

[15] Z. Dokur, T. Olmez, E. Yazgan, and O. K. Ersoy, "Detection of ECG waveforms by neural networks," Medical Engineering and Physics, 1997.

[16] S. Graja and J.-M. Boucher, "Hidden markov tree model applied to ECG delineation," IEEE Transactions on Instrumentation and Measurement, 2005.

[17] C. Li, C. Zheng, and C. Tai, "Detection of ECG characteristic points using wavelet transforms," IEEE Transactions on Biomedical Engineering, 1995.

[18] J. Martinez, R. Almeida, S. Olmos, A. Rocha, and P. Laguna, "A wavelet-based ECG delineator: evaluation on standard databases," IEEE Transactions on Biomedical Engineering, 2004.

[19] J. Willems, P. Arnaud, J. van Bemmel, R. Degani, P. Macfarlane, and C. Zywietz, "Common standards for quantitative electrocardiography: goals and main results. CSE working party." Methods of Information in Medicine, 1990

[20] IcyHeart European Project: www.icyheart-project.eu/

[21] A. Cohen and J. Kovacevic, "Wavelets: the mathematical background," Proceedings of the IEEE, 1996.

[22] L. Sornmo and P. Laguna, "Bioelectrical Signal Processing in Cardiac and Neurological Applications," Elsevier, 2005. 